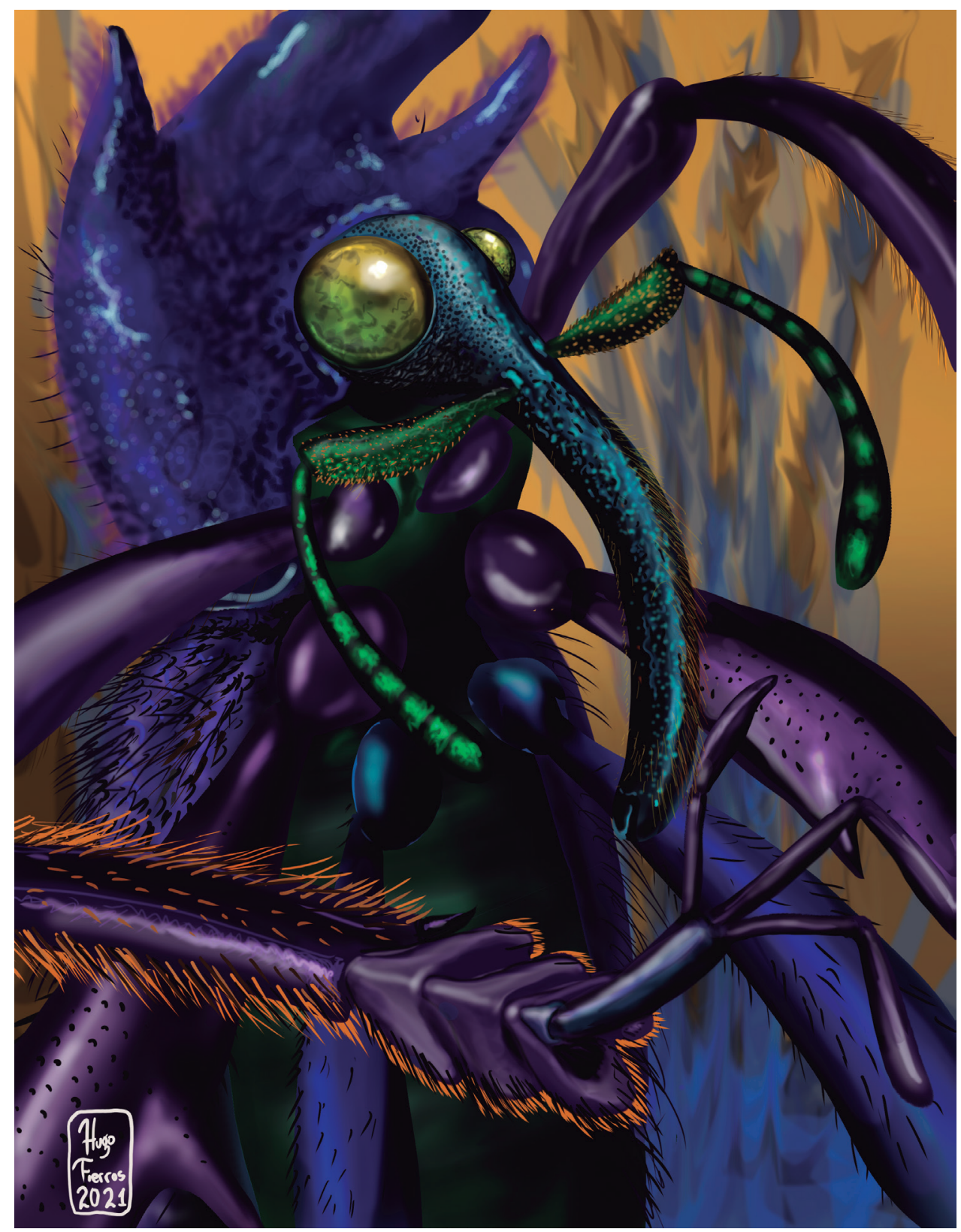

Dugesiana, Año 28, No. 2, (julio-diciembre, segundo semestre de 2021), es una publicación semestral, editada por la Universidad de Guadalajara, a través del Centro de Estudios en Zoología, por el Centro Universitario de Ciencias Biológicas y Agropecuarias. Camino Ramón Padilla Sánchez \# 2100, Nextipac, Zapopan, Jalisco, Tel. 37771150 ext. 33218, http://148.202.248.171/dugesiana/index.php/DUG/index, glenusmx@gmail.com. Editor responsable: José Luis Navarrete-Heredia. Reserva de Derechos al Uso Exclusivo 04-2009-062310115100-203, ISSN: 2007-9133, otorgados por el Instituto Nacional del Derecho de Autor. Responsable de la última actualización de este número: José Luis Navarrete-Heredia, Editor y Ana Laura González-Hernández, Asistente Editorial. Fecha de la última modificación 1 de julio de 2021, con un tiraje de un ejemplar.

Las opiniones expresadas por los autores no necesariamente reflejan la postura del editor de la publicación.

Queda estrictamente prohibida la reproducción total o parcial de los contenidos e imágenes de la publicación sin previa autorización de la Universidad de Guadalajara. 
Artículo

\title{
Plantas hospederas y localidades de colecta de Lepturinae (Coleoptera: Cerambycidae) en Chiapas, México
}

\section{Host plants and collection locations of Lepturinae (Coleoptera: Cerambycidae) in Chiapas, Mexico}

\author{
Carlos Joaquin Morales-Morales*1, Eduardo Aguilar-Astudillo ${ }^{2}$ y Reynerio A. Alonso-Bran ${ }^{3}$ \\ Universidad Autónoma de Chiapas, Facultad de Ciencias Agronómicas, Campus V, Carretera Ocozocoautla- \\ Villaflores, km 84, CP. 30470, Villaflores, Chiapas, México. Correspondencia carlosjoaquinm@yahoo.com.mx; \\ 'ORCID: htpps://orcid.org/0000-0001-6929-1502; ${ }^{2}$ ORCID: https://orcid.org/0000-0001-8057-8782; ${ }^{3}$ ORCID: \\ https://orcid.org/0000-0002-5959-0709
}

\begin{abstract}
RESUMEN
De la subfamilia Lepturinae (Cerambycidae) se revisaron 57 ejemplares, colectados sobre flores de Bucida macrostachya Standl. (Combretaceae) conocida en la región como cacho de toro, Hechtia schotii Baker ex Hemsley (Bromeliaceae), Jatropha curcas L. (Euphorbiaceae) piñón y Forsteronia spicata (Jacq.) G. Mey (Apocinaceae) bejucos leñosos, en cuatro municipios del estado de Chiapas. Se determinaron las especies: Choriolaus howdeni Giesbert and Wapes, 1999, Cyphonotida rostrata Sapes (Bates, 1872), Strangalia biannulata (Lynsley, 1935), Strangalia bicolorella Chemsak, 1969, Strangalia brachialis (Bates, 1885) Strangalia doyeni Chemsak \& Linsley, 1976, Strangalia melampus (Bates, 1885), Strangalia palaspina Chemsak, 1969, y Strangalia pectoralis (Bates, 1885), las cuales se encuentran depositadas en la colección entomológica de la Facultad de Ciencias Agronómicas Campus V, Villaflores $(\mathrm{CACH})$. De acuerdo con la literatura consultada la especie Cyphonotida rostrata pectorales (Bates) no está registrada para Chiapas por lo que estos datos representan los primeros registros.
\end{abstract}

Palabras clave: Revisado, Colecta, Hospedera, Distribución, Municipio

\section{ABSTRACT}

Of the Lepturinae (Cerambycidae) subfamily, 57 specimens were reviewed, collected on flowers of Bucida macrostachya Standl. (Combretaceae) known in the region as bull horn, Hechtia schotii Baker ex Hemsley (Bromeliaceae), Jatropha curcas L. (Euphorbiaceae) pinion and Forsteronia spicata (Jacq.) G. Mey (Apocinaceae) woody vines, in four municipalities of the state Chiapas. The species were determined: Choriolaus howdeni Giesbert and Wapes, 1999, Cyphonotida rostrata Hyphenated (Bates, 1872), Strangalia biannulata (Lynsley, 1935), Strangalia bicolorella Chemsak, 1969, Strangalia brachialis (Bates, 1885) Strangalia doyeni Chemsak \& Linsley, 1976, Strangalia melampus (Bates, 1885), Strangalia palaspina Chemsak, 1969, y Strangalia pectoralis (Bates, 1885), which are deposited in the Entomological Collection of the Faculty of Agronomic Sciences Campus V, Villaflores (CACH). According to the literature consulted, the species Cyphonotida rostrata paraspinal (Bates) is not registered for Chiapas, so these data represent the first records.

Keywords: Reviewed, Collection, Host, Distribution, Municipality.

Los insectos tienen hospederas específicas que le sirven para su alimentación, refugio y reproducción; dependiendo de las condiciones de desarrollo de la planta. En este sentido, en Chiapas, no existen reportes sobre la especificidad hacia sus plantas hospederas de los especímenes de la familia Cerambycidae, la cual puede ser muy variable; se conocen desde especies monófagas hasta especies altamente polífagas; se alimentan de especies vegetales de los grupos de las gimnospermas y angiospermas, como especies de los géneros Pinus, Abies, Larix, Picea, Quercurs, Salix, Carpinus, Amygdalus, Junglans, Prunus, Ceratonia, Ulmus, Platanus, Pistacea, Eucaliptus, Ficus, Nerium, Punica, Sorbus, Morus, Populus, Cercis, Cornus, Acacia, Genista, Colutea, Sisymbrium, Marrubium, Raphanus, Raphanistrum, Spartium, Sarothamnus, Calycotome, Cytisus, Alnus, Acer, Rosa, Ostrya, Carpinus, Crataegus, Betula y Castanea (Verdugo 2013) En el caso de las especies polífagas, éstas toman como hospederos solamente a uno o dos grupos de plantas como los géneros Ergates y Arhopalus, que están restringidos a coníferas; el género Phoracantha se reporta para Eucaliptos (Linsley 1959; Verdugo 2013). La distribución de los Cerambycidae varía significativamente con el tipo de vegetación, plantas hospederas potenciales y fuentes de alimentación (Vance et al. 2003); se tiene poco conocimiento sobre aspectos biológicos, hábitat y comportamiento, especialmente de las plantas hospederas (Zuñiga-Reynoso 2013). Diversos estudios y catálogos de plantas hospederas de Cerambycidae han sido publicados en los últimos años, como los cerambícidos de Sierra Nevada, España (Tavakilian et al. 1997; Monné 2004, 2005a, b; Machado et al. 2009; McRae et al. 2012; Verdugo 2013); sin embargo, aún se desconocen muchas de las especies de plantas asociadas a diferentes especies de escarabajos longicornios como es el caso de la subfamilia Lepturinae de los cuales hay escasa información, como los publicados por Monné $(2004,2005)$ y Verdugo (2013), Los integrantes de la subfamilia Lepturinae se caracterizan por presentar el cuerpo elongado, esbelto, con patas delgadas 
y alargadas, las antenas no sobrepasan la longitud del cuerpo; muchas de las especies que integran este grupo mimetizan a los himenópteros especialmente de la Familia Vespidae. En México se conocen 81 especies (Noguera 2014) de las cuales 23 están reportadas para el estado de Chiapas (Monné 2016). En este trabajo se dan a conocer las plantas hospederas y nuevas localidades de las especies de la subfamilia Lepturinae en el estado de Chiapas.

\section{MATERIALES Y MÉTODOS}

Se realizaron colectas en diferentes localidades del estado de Chiapas, utilizando una red entomológica aérea y de manera manual, cuando los especímenes se encontraban posados o se alimentaban de las flores de las plantas. Para la determinación a especie, conocimiento de la distribución geográfica y plantas hospederas se consultaron las obras de Noguera y Chemsak (1996), Monné y Hovore (2001, 2005), Monné (2006), Maes et al. (2010), Monné y Bezark (2012), Bezark y Monné (2013), Gutiérrez y Noguera (2015). Los especímenes citados en este trabajo se encuentran depositados en la Colección Entomológica, de la Facultad de Ciencias Agronómicas, Campus V, de la Universidad Autónoma de Chiapas (CACH).

La determinación especifica de las plantas hospederas donde se colectaron los lepturinos, fue realizado por el Biol. Eduardo Palacios Espinosa, investigador del Jardín Botánico Faustino Miranda, ubicado en la ciudad de Tuxtla Gutiérrez, Chiapas, las cuales quedaron depositados en el herbario de dicha institución.

\section{RESULTADOS}

Se revisaron 57 ejemplares de la Subfamila Lepturinae, de los cuales se determinaron nueve especies, que tienen como hospederos a siete especies de plantas que se incluyen en seis familias botánicas (Cuadro 1).

Las especies revisadas de la subfamilia Lepturinae reportados en este trabajo fueron colectados en cuatro localidades de cuatro municipios del estado de Chiapas; encontrando mayor abundancia de especies y de individuos en la localidad de El Aguacero, del municipio de Ocozocoautla, Chiapas (Cuadro 2); además, la mayoría de las especies se colectaron durante el mes de junio.

De acuerdo a la distribución conocida de las especies de leptorinos se tiene que Choriolaus howdeni Giesbert \& Wappes, 1999, se encuentra reportada para El Aguacero, Ocozocoautla, Chiapas, México. Cyphonotida rostrata (Bates, 1872), se cita para México para los estados de Baja California y Jalisco. Se amplía su registro para los estados de Guerrero, Nayarit, Sinaloa y Veracruz; Además, para el estado de Oaxaca; y para los Estados Unidos de América (Texas), Guatemala, El Salvador, Nicaragua, Panamá, Guyana, Ecuador, Brasil, Colombia, Guyana, Bolivia, Ecuador y Brasil; esta especie acude a las flores de Croton sp. (Euphorbiaceae), Coccoloba sp. (Polygonaceae) y Bumelia celastrina Kunth (Sapotaceae). Las especies Strangalia biannulata (Lynsley, 1935), se encuentra distribuida en los estados del Distrito Federal, Chiapas (Villaflores: San Ramón), Guerrero, México, Michoacán, Morelos y Oaxaca. Strangalia bicolorella (Chemsak, 1969) se reporta para las localidades de los estados de Chiapas (Ocozocoautla: El Aguacero), Colima, Jalisco (Chamela) y Sinaloa. Strangalia brachialis (Bates, 1885) se encuentra en los estados de Chiapas, Oaxaca, Quintana Roo y Veracruz; extendiéndose a Belice. Strangalia doyeni Chemsak \& Linsley, 1976 se cita para Chiapas (Ocozocoautla: El Aguacero), Jalisco, Nayarit, Morelos y Oaxaca; además de Guatemala. Strangalia melampus (Bates, 1885), indican que esta especie se encuentra en Chiapas (Oxchuc y El Porvenir) y en Guatemala. Strangalia palaspina (Chemsak, 1969) esta especie presenta amplia distribución en México se cita para los estados de Chiapas (El Aguacero), Jalisco (Chamela), Nayarit y Sinaloa; además de Guatemala y Strangalia pectoralis (Bates, 1885) se reporta para los estados de Chiapas, Oaxaca y Veracruz; además de Belice (Chemsak y Noguera 1993; Noguera y Chemsak 1996; Toledo et al. 2002; Hovore 2006; Monné 2006; Noguera et al. 2007; Morales-Morales et al. 2012; Monne 2013; Bezark y Monné 2013; Gutiérrez y Noguera, 2015; Monné, 2020).

\section{DISCUSIÓN}

De las cinco especies de lepturinos citados por Toledo et al (2002) para el Aguacero (Ocozocoautla: Chiapas) se reportan en este trabajo a Choriolaus howdeni Giesbert y Wappes, Strangalia bicolorella Chemsak, Strangalia doyeni Chemsak y Linsley, y Strangalia palaspina Chemsak, los cuales fueron colectados en la misma localidad durante los meses de junio y julio, que es la etapa de floración de las plantas Bucida macrostachya Standl., Hechtia schotii Baker ex Hemsley, Jatropha curcas L. y Forsteronia spicata (Jacq.) G. Mey. que fue el periodo de mayor abundancia de ejemplares colectados.

Las especies Strangalia biannulata (Lynsley) y Strangalia brachialis (Bates), fueron reportados por Morales-Morales et al. (2012) para los municipios de Villacorzo y Villaflores respectivamente; la especie Strangalia melampus (Bates) es reportada por primera vez para Chiapas por Gutiérrez y Noguera (2015) colectadas en los municipios de Oxchuc y el Porvenir; con los datos proporcionados en este trabajo se extiende el rango de distribución para el estado de Chiapas.

Se reporta para Chiapas a Choriolaus howdeni Giesbert y Wappes, Strangalia doyeni Chemsak y Linsley, $S$. pectoralis (Bates) sin especificar localidades de colecta (Bezark y Monné, 2013). La especie Cyphonotida rostrata rostrata (Bates) fue reportado por Monné $(2013,2020)$ para los estados de Baja California, Guerrero, Jalisco, Oaxaca, Sinaloa y Veracruz; siendo estos datos los primeros registros para Chiapas. Monné (2020) reporta que esta especie acude a las flores de Croton sp. (Euphorbiaceae), Coccoloba sp. (Polygonaceae) y Bumelia celastrina Kunth (Sapotaceae); Las especies C. howdeni, C. rostrata, S. doyeni y S. pectoralis; fueron colectados en las flores de Bucida macrostachya Standl. (Combretaceae); el cual se considera como un indicador que esta especie vegetal es más atractiva para estas especies de lepturinos. 


\section{LITERATURA CITADA}

Bezark, L. G. and M. A. Monné. 2013. Checklist of the Oxypeltidae, Vesperidae, Disteniidae and Cerambycidae. (Coleoptera) of the Western Hemisphere. Disponible en línea: http://plant.cdfa.ca.gov/byciddb/checklists/ WestHemiCerambycidae2013.pdf. Fecha de consulta: 8 de agosto de 2018.

Chemsak A. J. and F. A. Noguera. 1993. Annotated checklist of the Carambycidae of the Estación de Biologia Chamela, Jalisco, Mexico (Coleoptera), with descriptions of nex genera and species. Folia Entomológica Mexicana, (89): 55 - 102.

Gutiérrez, N. and F. A. Noguera. 2015. New distributional records of Cerambycidae (Coleoptera) from Mexico. The Pan-Pacific Entomologist, 91(2), 135-147.

Hovore, F. T. 2006. The Cerambycidae (Coleoptera) of Guatemala, p. 363-378. En: Biodiversidad de Guatemala. E. Cano (Ed.). Universidad del Valle de Guatemala, Guatemala.

Linsley, E. G. 1959. Ecology of Cerambycidae. Annual Review of Entomology (4):99-138.

Machado, S., V., J.P. Botero, A. Carelli, M. Cupello, Y. Hingrid Q. and M.V. P. Simões. 2012. Host plants of Cerambycidae and Vesperidae (Coleoptera, Chrysomeloidea) from South America. Revista Brasileira de Entomologia, 56(2): 186-198. (Revisar cita)

MacRae C., T., L.G. Bezark and I. Swift. 2012. Notes on distribution and host plants of Cerambycidae (Coleoptera) from southern Mexico. The Pan-Pacific Entomologist, (88):173-187.

Maes, J. M., E. Van den Berghe, D. Dauber, A. Audureau, E. Nearns, F. Skilman, D. Heffern and M. Monné. 2010. Catalogo Ilustrado de los Cerambycidae (Coleoptera) de Nicaragua. Parte III-Lepturinae. In memoriam Frank T. Hovore. $31 \mathrm{p}$.

Monné M. A. 2006. Catalogue of the Cerambycidae (Coleoptera) of the Neotropical Region. Part III. Subfamilies Parandrinae, Prioninae, Anoplodermatinae, Aseminae, Spondylidinae, Lepturinae, Oxypeltinae, and addenda to the Cerambycinae and Lamiinae. Zootaxa, 1212: 1-244.

Monné M. A. and F. T. Hovore. 2001. Checklist of the Cerambycidae and Disteniidae (Coleoptera) of the Western Hemisphere. Disponible en línea: http:// cerambycidae.cl/bibliografia/Checklist $\% 20$ of $\% 20$ the $\% 20$ Cerambycidae $\% 20$ and $\% 20$ Disteniidae $\% 20$ of $\% 20$ the $\% 20$ Western $\% 20 \mathrm{Hem}$ is phere $\% 20$ - $20 \mathrm{Part} \% 202, \% 20 \mathrm{Lami}$ ina $\% 20$ through $\% 20$ Disteniinae\%20(2001).pdf. Fecha de consulta: 2 mayo 2020.

Monné M. A. and F. T. Hovore. 2005. Checklist of the Cerambycidae of the Western Hemisphere. Disponible en línea: http://www.cerambycoidea.com/papersEl. asp? $\mathrm{Id}=\&$ Lett=M\&NPag=5. Fecha de consulta: 10 de abril 2020.
Monné, M. A. 2004. Catalogue of the Neotropical Cerambycidae (Coleoptera) with known host plant - Part VI: Host plant index. Publicações Avulsas do Museu Nacional, Rio de Janeiro, (100): 1-95.

Monné, M. A. 2005a. Catalogue of the Cerambycidae (Coleoptera) of the Neotropical region. Part I. Subfamily Cerambycinae. Zootaxa, (946):1-765.

Monné, M. A. 2005b. Catalogue of the Cerambycidae (Coleoptera) of the Neotropical region. Part II. Subfamily Lamiinae. Zootaxa, (1023):1-759.

Monné, M. A. 2013. Catalogue of the Cerambycidae (Coleoptera) of the Neotropical Region. Part III. Subfamilies Lepturinae, Necydalinae, Parandrinae, Prioninae, Spondylidinae and Families Oxypeltidae, Vesperidae and Disteniidae. Disponible en línea: https://www.researchgate.net/publication/286016156_ Catalogue_of_the_Cerambycidae_Coleoptera_of_the Neotropical_Region_Part_II_Subfamily_Lamiinae. Fecha de consulta: 15 de marzo 2020.

Monné, M. A. 2016. Catalogue of the Cerambycidae (Coleoptera) of the Neotropical Region. Part III. Subfamilies Lepturinae, Necydalinae, Parandrinae, Prioninae, Spondylidinae and Families Oxypeltidae, Vesperidae and Disteniidae. Disponible en línea: http:// cerambycidae.miza-ucv.org.ve/es/node/231. Fecha de consulta: 17 de marzo de 2019.

Monné, M. A. 2020. Catalogue of the Cerambycidae (Coleoptera) of the Neotropical Region. Part III. Subfamilies Lepturinae, Necydalinae, Parandrinae, Prioninae, Spondylidinae and Families Oxypeltidae, Vesperidae and Disteniidae. Disponible en línea: https:// cerambycids.com/catalog. Fecha de consulta: 8 de junio de 2020.

Monné, M. A. and L. G. Bezark. 2012. Cerambycidae: Cerambycinae species from México. In: Cerambycidae de México, Cerambycinae. Disponible en linea: http:// www.coleopteraneotropical.org/paginas/2_PAISES/ Mexico/Cerambycidae/cerambycinae-mex.html. Fecha de consulta: 23 de abril de 2019 .

Monné, M.A. 2002. Catalogue of the Neotropical Cerambycidae (Coleoptera) with known host plant-part V: Subfamilies Prioninae, Parandrinae, Oxypeltinae, Anoplodermatinae, Aseminae and Lepturinae. Publicações Avulsas do Museu Nacional, (96):3-70. Disponible en: https://biblat.unam.mx/es/revista/ publicacoes-avulsas-do-museu-nacional/articulo/ catalogue-of-the-neotropical-cerambycidae-coleopterawith-known-host-plant-part-v-subfamilies-prioninaeparandrinae-oxypeltinae-anoplodermatinae-aseminaeand-lepturinae. Fecha de Consulta: 25 abril 2020.

Morales-Morales, C. J., E. Aguilar-Astudillo, M. A. Rosales-Esquinca, R. R. Quiroga-Madrigal, R. A. Alonso-Bran y R. C. Gutiérrez-Hernández. 2012.. Cerambícidos (Coleoptera, Cerambycidae) asociados al piñón (Jatropha curcas L.), en cinco municipios de la Depresión Central de Chiapas, México. Revista Biota 
Colombiana, 13(1): 35-46.

Noguera, F. A. y J. A. Chemsak. 1996. Cerambycidae (Coleoptera), pp. 381-409. In: J. E. Llorente-Bousquets, A. N. García-Aldrete \& E. González-Soriano (Eds.). Biodiversidad, taxonomía y biogeografía de artrópodos de México: Hacia una síntesis de su conocimiento. Instituto de Biología, UNAM, México, D. F.

Noguera, F. A., Chemsak, J. A., Zaragoza-Caballero, S., Rodríguez-Palafox, A., Ramírez-García, E., GonzálezSoriano, E \& Ayala, R. 2007. A faunal study of Cerambycidae (Coleoptera) from one region with tropical dry forest in México: San Buenaventura, Jalisco. The Pan-Pacific Entomologist 83(4), 296-314.

Noguera. F. A. 2014. Biodiversidad de Cerambycidae (Coleoptera) en México. Revista Mexicana de Biodiversidad, (85): 290-297.

Tavakilian, G. L., A. Berkov, B. Meurer-Grimes and S. Mori. 1997. Neotropical tree species and their faunas of xylophagous longicorns (Coleoptera: Cerambycidae) in French Guiana. Botanical Review, (63): 304-355.
Toledo, V. H., F. A. Noguera, J. A. Chemsak, F. T. Hovore and E. F. Giesbert. 2002. The cerambycid fauna of the tropical dry forest of "El Aguacero," Chiapas, México (Coleoptera: Cerambycidae). The Coleopterists Bulletin 56(4):515-532.

Vance, C. C., K. R. Kirby, J. R. Malcolm, and S. M. Smith. 2003. Community composition of longhorned beetles (Coleoptera: Cerambycidae) in the canopy and understorey of sugar maple and white pine stands in south-central Ontario. Environmental Entomology, (32): 1066-1074.

Verdugo P., A. 2013. Los cerambícidos (Coleoptera: Cerambycidae). (pp. 81-99). En: Ruano, F., M., Tierno de Figueroa y A. Tinaut (Eds.) Los Insectos de Sierra Nevada. 200 años de historia. Asociación española de Entomología, León.

Zúñiga-Reynoso, A. 2013. Revisión de los Cerambycidae (Coleoptera) de la región de Magallanes, lista ilustrada. Chile. Anales Instituto de Patagonia, 41(1):53-59.

Recibido: 11 febrero 2021

Aceptado: 27 abril 2021

Cuadro 1. Especies de Lepturinae depositadas en la colección Entomológica y sus plantas hospederas.

\begin{tabular}{|c|c|c|c|c|}
\hline Familia & Especie & Hospedero & Familia & Cita \\
\hline \multirow[t]{13}{*}{$\begin{array}{l}\text { Cerambycidae } \\
\text { Lepturinae }\end{array}$} & \multirow[t]{2}{*}{$\begin{array}{l}\text { Choriolaus howdeni } \\
\text { Giesbert \& Wappes, } 2000\end{array}$} & Bucida macrostachya Standl & Combretaceae & $\begin{array}{l}\text { Toledo et al. } \\
\text { (2002) } \\
\text { Monné, 2006) }\end{array}$ \\
\hline & & $\begin{array}{l}\text { *Hechtia schotii Baker ex } \\
\text { Hemsley }\end{array}$ & Bromeliacea & \\
\hline & \multirow{3}{*}{$\begin{array}{l}\text { Cyphonotida rostrata (Bates, } \\
1872 \text { ) }\end{array}$} & Croton $\mathrm{sp}$ & Euphorbiaceae & \multirow[t]{3}{*}{ Monné (2020) } \\
\hline & & Coccoloba sp. & Polygonaceae & \\
\hline & & Bumelia celastrina Kunth & Sapotaceae & \\
\hline & $\begin{array}{l}\text { Strangalia biannulata } \\
\text { (Lynsley, 1935) }\end{array}$ & Jatropha curcas L. & Euphorbiaceae & $\begin{array}{l}\text { Morales-Morales } \\
\text { et al. } 2012\end{array}$ \\
\hline & $\begin{array}{l}\text { Strangalia bicolorella } \\
\text { Chemsak, } 1969\end{array}$ & $\begin{array}{l}\text { *Forsteronia spicata (Jacq.) } \\
\text { G. Mey. }\end{array}$ & Apocinaceae & \\
\hline & $\begin{array}{l}\text { Strangalia brachialis (Bates, } \\
1885 \text { ) }\end{array}$ & Jatropha curcas $\mathrm{L}$. & Euphorbiaceae & $\begin{array}{l}\text { Morales-Morales } \\
\text { et al. } 2012\end{array}$ \\
\hline & \multirow[t]{2}{*}{$\begin{array}{l}\text { Strangalia doyeni Chemsak } \\
\text { \& Linsley, } 1976\end{array}$} & $\begin{array}{l}\text { *Bucida macrostachya } \\
\text { Standl. }\end{array}$ & Combretaceae & \\
\hline & & $\begin{array}{l}\text { Forsteronia spicata (Jacq.) } \\
\text { G. Mey. }\end{array}$ & Apocinaceae & \\
\hline & $\begin{array}{l}\text { Strangalia melampus (Bates, } \\
1885 \text { ) }\end{array}$ & Jatropha curcas L. & Euphorbiaceae & $\begin{array}{l}\text { Morales-Morales } \\
\text { et al. } 2012\end{array}$ \\
\hline & $\begin{array}{l}\text { Strangalia palaspina } \\
\text { Chemsak, } 1969\end{array}$ & $\begin{array}{l}\text { *Hechtia schotii Baker ex } \\
\text { Hemsley }\end{array}$ & Bromeliacea & \\
\hline & $\begin{array}{l}\text { Strangalia pectoralis (Bates, } \\
1885 \text { ) }\end{array}$ & $\begin{array}{l}\text { * Bucida macrostachya } \\
\text { Standl. }\end{array}$ & Combretaceae & \\
\hline
\end{tabular}


Cuadro 2. Lugares de colecta y número de individuos de las especies de Lepturinae (Cerambycidae).

\begin{tabular}{|c|c|c|c|c|}
\hline Especie & Localidad & Municipio & $\begin{array}{c}\text { No. } \\
\text { ejemplares }\end{array}$ & Fecha colecta \\
\hline \multirow{5}{*}{$\begin{array}{l}\text { Choriolaus howdeni } \\
\text { Giesbert \& Wappes, } 2000\end{array}$} & El Aguacero & Ocozocoautla & 1 & 7/junio/1993 \\
\hline & & & 8 & $18 /$ junio/2000 \\
\hline & & & 1 & 24/junio/2001 \\
\hline & & & 4 & 7/julio/2002 \\
\hline & & & 8 & 14/junio/2003 \\
\hline \multirow{3}{*}{$\begin{array}{l}\text { Cyphonotida rostrata } \\
\text { (Bates, 1872) }\end{array}$} & El Aguacero & Ocozocoautla & 2 & $18 /$ junio/2000 \\
\hline & & & 1 & 24/junio/2000 \\
\hline & San Ramón & Villaflores & 1 & $20 /$ agosto/2011 \\
\hline $\begin{array}{l}\text { Strangalia biannulata } \\
\text { (Lynsley, 1935) }\end{array}$ & San Ramón & Villaflores & 1 & 11/junio/2011 \\
\hline \multirow{3}{*}{$\begin{array}{l}\text { Strangalia bicolorella } \\
\text { Chemsak, } 1969\end{array}$} & El Aguacero & Ocozocoautla & 2 & $24 /$ junio/2000 \\
\hline & & & 1 & 17/junio/2002 \\
\hline & & & 1 & 8/julio/2002 \\
\hline \multirow{3}{*}{$\begin{array}{l}\text { Strangalia brachialis } \\
\text { (Bates, 1885) }\end{array}$} & Rancho San Ignacio & Villacorzo & 1 & 28/mayo/2009 \\
\hline & San Ramón & Villaflores & 1 & $11 /$ junio/2011 \\
\hline & & & 1 & 25/junio/2011 \\
\hline \multirow{4}{*}{$\begin{array}{l}\text { Strangalia doyeni } \\
\text { Chemsak \& Linsley, } \\
1976\end{array}$} & El Aguacero & Ocozocoautla & 2 & 18/junio/2000 \\
\hline & & & 2 & 24/junio/2000 \\
\hline & & & 1 & 16/junio/2001 \\
\hline & & & 1 & 14/junio/2003 \\
\hline \multirow{2}{*}{$\begin{array}{l}\text { Strangalia melampus } \\
\text { (Bates, 1885) }\end{array}$} & San Ramón & Villaflores & 1 & 19/junio/2014 \\
\hline & & & 1. & 2/julio/2014 \\
\hline \multirow{2}{*}{$\begin{array}{l}\text { Strangalia palaspina } \\
\text { Chemsak, } 1969\end{array}$} & El Aguacero & Ocozocoautla & 2 & $24 /$ junio/2000 \\
\hline & & & 2 & 14/junio/2003 \\
\hline \multirow{2}{*}{$\begin{array}{l}\text { Strangalia pectoralis } \\
\text { (Bates, } 1885 \text { ) }\end{array}$} & La Pilar & Cintalapa & 7 & $13 /$ abril $/ 2001$ \\
\hline & & & 4 & 28/marzo/2002 \\
\hline Total & 4 & 4 & 57 & \\
\hline
\end{tabular}

